\title{
Dental Bleaching a Case Report Presenting What Science and Clinical Evidence Shows in Terms of Result, Safety, Comfort and Durability
}

\author{
Andreas Raphael Ribas Koren*1 and Renato Miotto Palo ${ }^{2}$ \\ ${ }^{1}$ Prosthodoncist, Brazil
}

${ }^{2}$ Endodontics postgraduate program coordinator, Thum Institute, Brazil

Received: February 01, 2018; Published: February 09, 2018

*Corresponding author: Andreas Raphael Ribas Koren, Prosthodoncist, Private practice: RuaGroenlândia, 183 Jd. America, Sao Paulo, SP, Brazil, Email: andreaskoren@hotmail.com

Abstract

The aim of this report is to describe two clinical cases of dental bleaching, both using CP $10 \%$ but in different biological teeth structuresyoung and older enamel and dentin. In both cases the "at home/overnight bleaching" was used as preferred technique, such as the shade guide Vita Classic Scale Vita, Zahnfabrik, Sackingen, Germany to color shade changing control. Case 1 reached a very satisfying result in a regular term, despite the initial condition. Case 2 reached a very satisfying result as well, but in a short term because of the patient's age and his dental favorable condition. This report shows how safe and predictable a dental bleaching can be, and what technique is more reliable to reach the expected and long lasting result, with comfort and no side effects.

\section{Introduction}

A beautiful smile is everybody's desire nowadays. Not just perfect shapes and sizes that makes a smile look beautiful and healthy, but also the color. Tooth color reveals many things such as people's oral health, good and bad habits and their age. And everyone wants to look young and well maintained. Tooth whitening became one of the most wanted and performed cosmetic dentistry procedures. Although is treatment is safe and scientifically proven, some precautions must be taken, such as looking for a qualified professional. There are many whitening products available on the market. Each one was developed for specific scenarios, since each and every patient is different from another. Cosmetic dentistry has developed ways and techniques to change teeth color, making the treatment comfortable, predicable, painless and achieving long lasting results. The chemical substance used in this procedure consists of car amide peroxide (CP) or hydrogen peroxide (HP). Both are presented as gel in different concentrations (1). Basically, there are two whitening techniques scientifically supported [1]. We have the "in-office bleaching" and the "at home/overnight bleaching". The "in-office bleaching" is performed only by a dentist at the dental office, and uses a high concentration hydrogen peroxide gel (35\%), which may cause slight, moderate or even severe dental sensitivity during the procedure and post operative, and their long term result is still questionable (2).
And the "at home/overnight bleaching" is a technique in which the patient applies the product inside a pre-fabricated silicone tray and fits it in his/her teeth. Depending on the CP or HP concentration, the treatment is performed overnight-gel concentration between $5 \%$ (HP) and 10\% (CP) - or during the day-gel concentration between $15 \%$ and $21 \%$, CP only (3). The first step to begin any cosmetic/ prosthetic procedures is dental bleaching. There are two different ways to follow up color shade changing along the treatment. The most used in day-by-day clinic is the shade guide. The other technique is using a spectrophotometer measurement, usually used in laboratorial experiments $(2,4)$. Tooth sensitivity during a bleaching treatment is classified in four stages, according to Marson none, slight, moderate and severe. A condition can be considered normal when the patient feels none or slight sensitivity for a well succeed treatment [2]. When the sensitivity is moderate or severe, the treatment must be immediately suspended and the doctor must review what is happening. Root exposure, gingival retraction, dentin exposure, broken filling and even high concentration of the HP or CP used can be the responsible issue of the sensitivity.

\section{Clinical Report}

\section{Case 1}

Patient PB, male, 57 years old, Caucasian, non-smoking, favorable and stable oral/periodontal health, with no previous 
record of dental bleaching. The patient presents some cosmetic needs, like filling replacements and anterior teeth improvement, which was planned to do after whitening. A root canal on tooth 31 was previously related as a color complication. In that case a different protocol was applied only to this tooth after dental bleaching technique to match its color to the other teeth. The step one is the color mapping [3,4]. For a better accuracy, photography is used as a fantastic resource to control the color shade progression, as we can see at the following images (Figures 1-3). It's very important to see the smile extension (dental exposure and mouth opening) to understand how far the cosmetic/prosthetic study should go to deliver an expected result. Patients with wider smiles, showing all their teeth will be pickier at the final result. The color mapping must be precise, not just on the upper front tooth but also on the lower and back tooth-premolars and molars (Figures 4-6). For a natural and harmonic result, the dental bleaching must be homogeneous. Despite gingival retractions and root exposure, the initial anamnesis indicates sensitivity absence to heat, cold and mechanical stimulus. Considering all these information and the patient's age, it is possible to predict lower or non-sensitivity symptoms during the treatment [5].

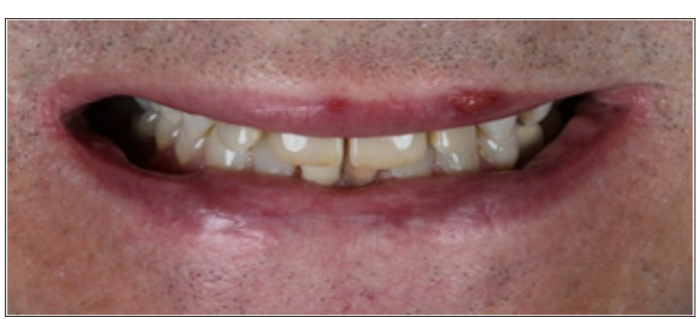

Figure 1: Case 1 - original smile.

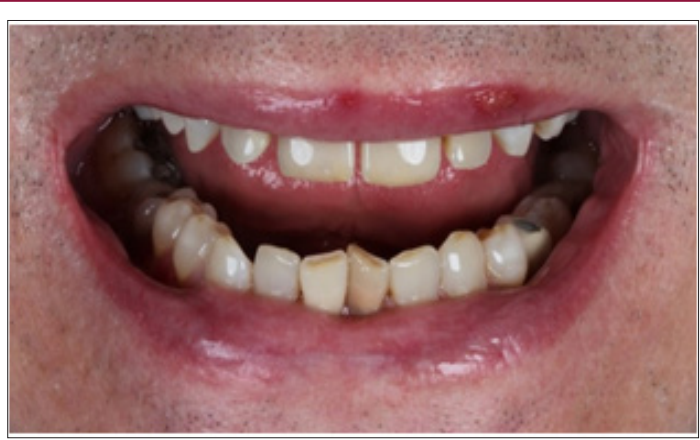

Figure 2: Case 1 - original smile - inferior view.

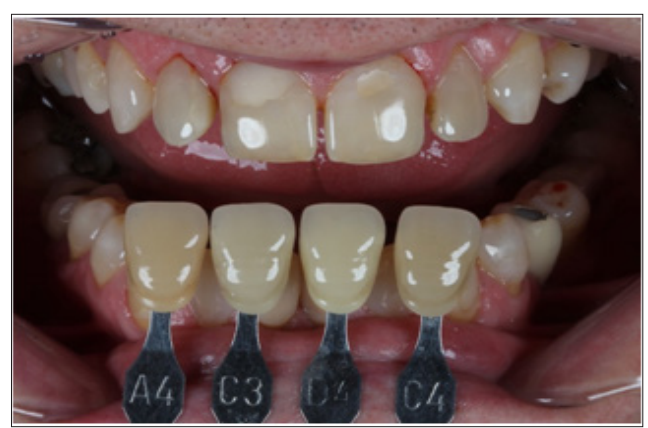

Figure 3: Initial shade mapping - anterior superior take.

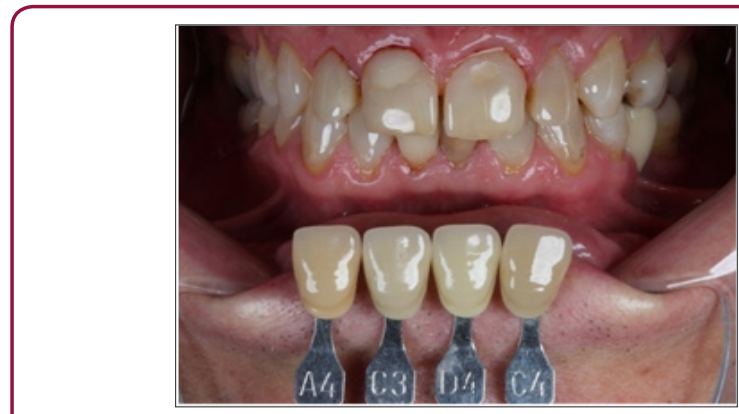

Figure 4: Initial shade mapping - overview take.

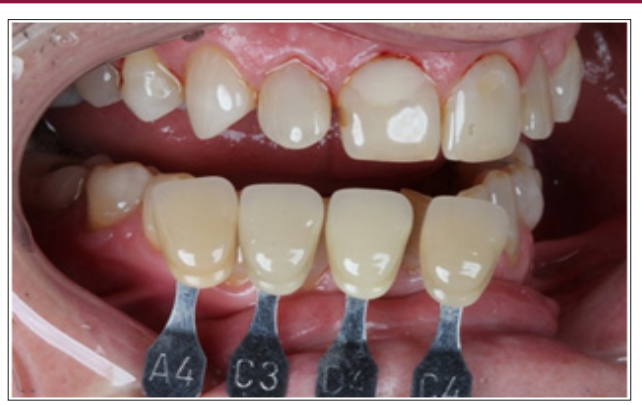

Figure 5: Initial shade mapping-right sups Ant Take.

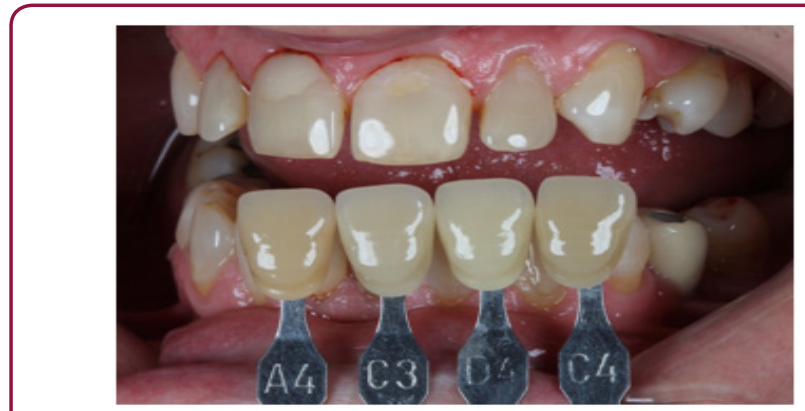

Figure 6: Initial shade mapping-left sup ant Take.

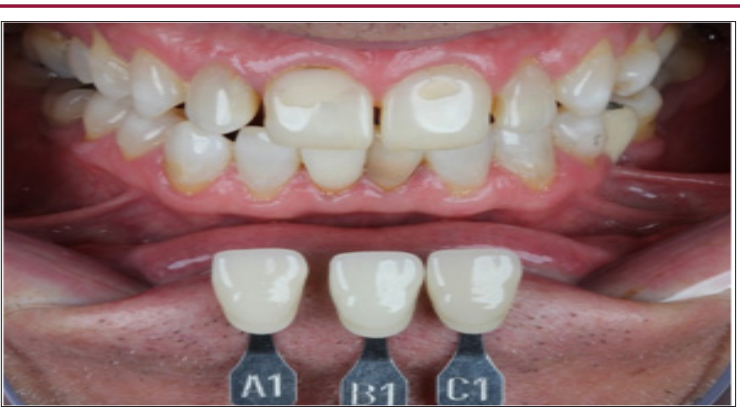

Figure 7: Final shade mapping - overview take.

The CP 10\% (Opalescence 10\%, UltradentInc, Salt Lake City, USA) was used as a "gold standard" elected material. It was used overnight, at least 6 hours per night. The treatment lasted 20 uninterrupted days. On day 10 a follow-up was made. No sensitivity signs were related, normal and healthy gums was related. On day 20 the patient came for follow up and photos. Comparing the initial shade to the final shade, the teeth whitening reached 3shades - B4 to C1 (Figures 3-7). The final result was positive, as can be seen at the images (Figures 7-9). After dental bleaching, some details unseen by the patient are now visible, like old veneers, crowns and 
fillings. But as can be seen on before/after images the result was successfully achieved (Figures $10 \& 11$ ). Now, the second part of the treatment - cosmetic/prosthetic dentistry - is able to begin to correct and improve all these details.

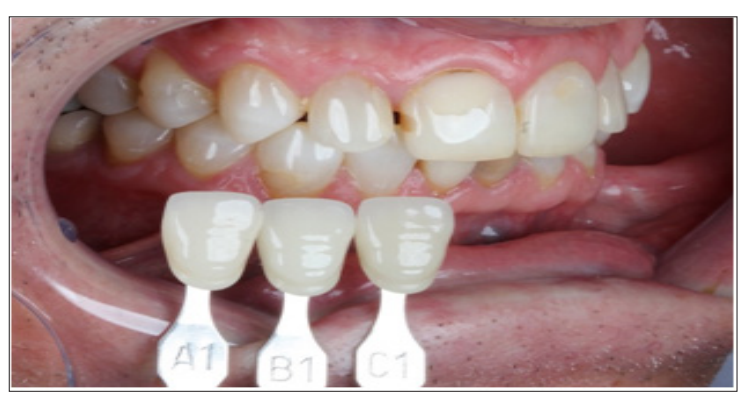

Figure 8: Final shade mapping - right posterior take.

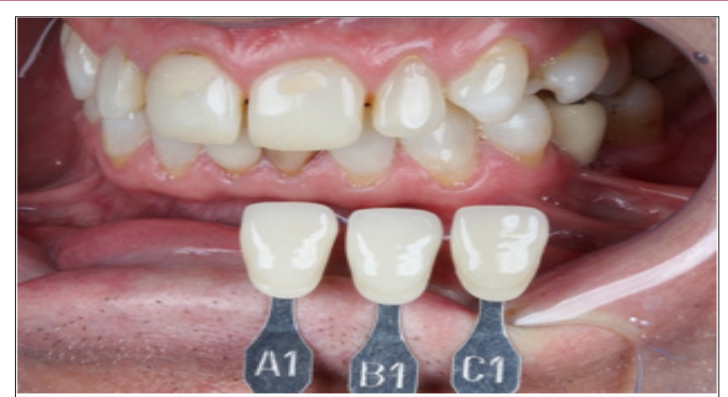

Figure 9: Final shade mapping - left posterior take.

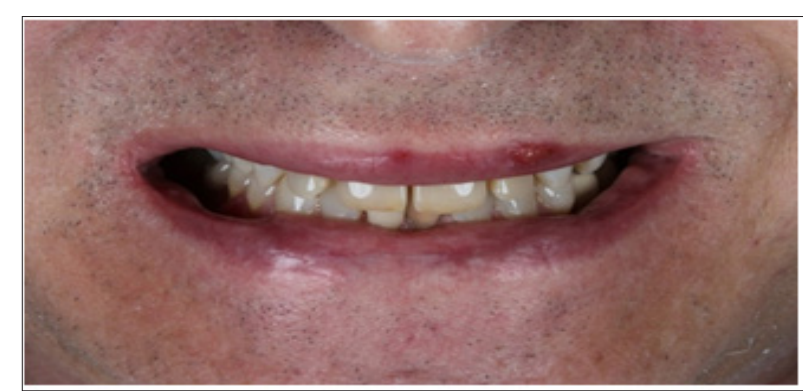

Figure 10: Case 1 - initial.

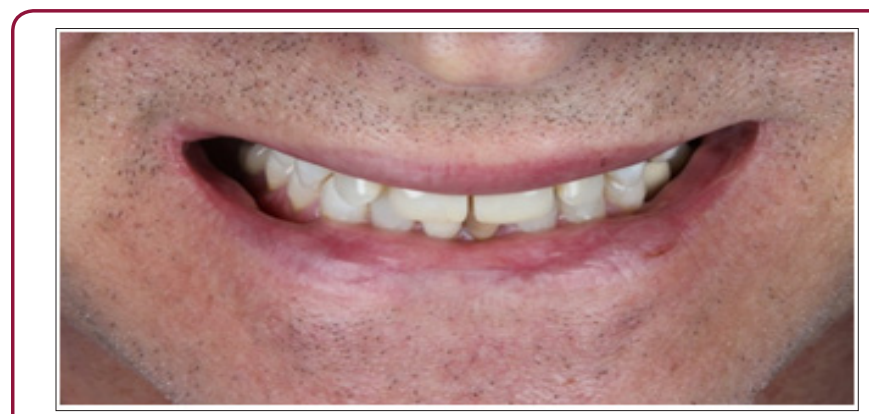

Figure 11: Case 1 - final result.

\section{Case 2}

Patient ED, male 32 years old, ginger, non-smoking, favorable and stable oral/gingival health, with no previous record of dental bleaching, with no cosmetic/prosthetic needs. Came to the office for oral maintenance and dental bleaching. After clinical exams and initial photos - photo mapping - a minimum root exposure was noticed on tooth 45 [6]. The patient does not present any dental sensitivity with cold, heat or mechanical stimulus. Analyzing the teeth color, a dark yellowish shade can be noticed especially in his canines and premolars, a condition that doesn't match his age (Figures 12-16). The CP 10\% (Opalescence 10\%, UltradentInc, Salt Lake City, USA) was used as a "gold standard" elected material. The overnight technique was performed, at least 6 hours per night. The treatment lasted 12 uninterrupted days. On day 12 the patient came for follow up session. The teeth response was fast and favorable due its challenging initial color (Figures 17-20). A slight sensitivity was related between day 3 and 5 in the first morning hours only. Comparing the initial shade to the final shade, the teeth whitening reached 4 shades - B4 to lighter than B1 - transcending the classic shade guide in all front teeth (Figures 21-23). Now, comparing the before/after images, it can be concluded that the expected result was successfully achieved (Figures $24 \& 25$ ).

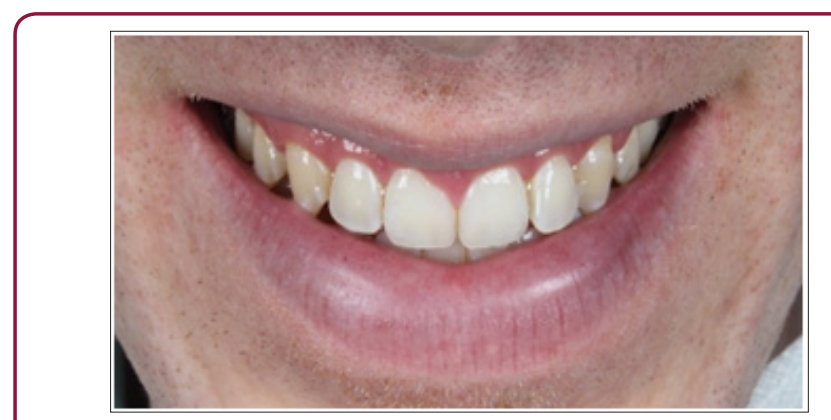

Figure 12: Case 2 - original smile.

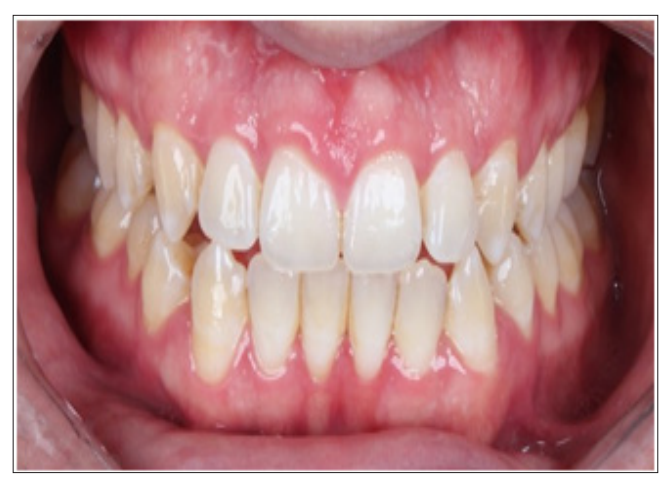

Figure 13: Case 2 - original smile overview.

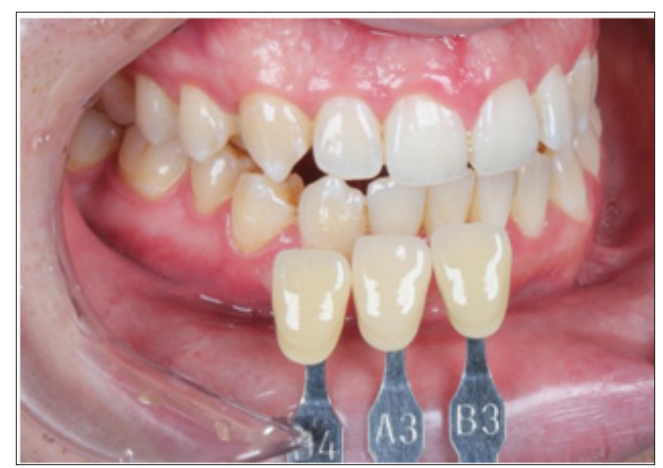

Figure 14: Initial shade mapping - right posterior take. 


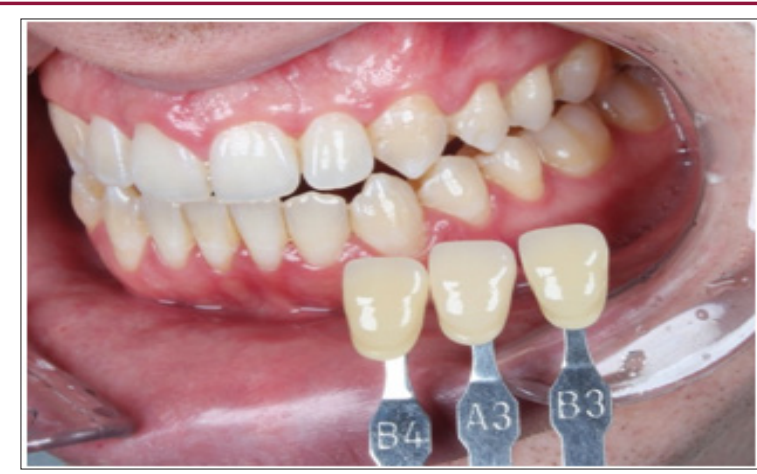

Figure 15: Initial shade mapping - left posterior take.

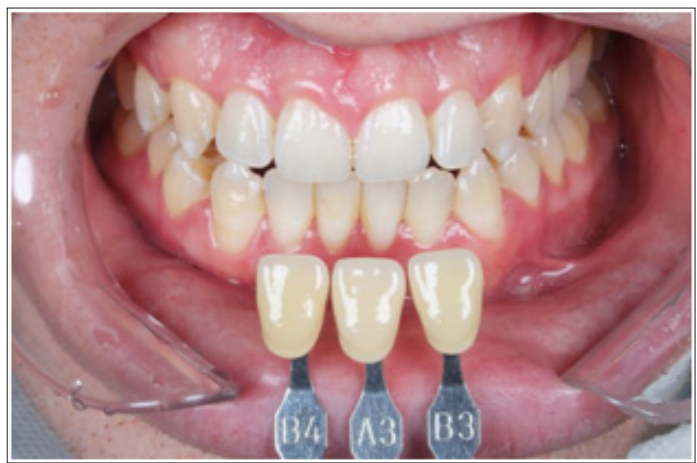

Figure 16: Initial shade mapping - overview take.

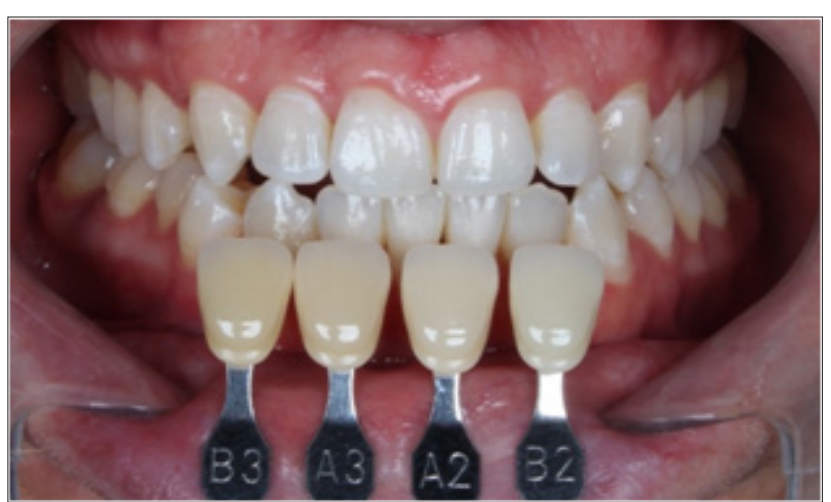

Figure 17: Final shade mapping - comparison to the sup Initial shade.

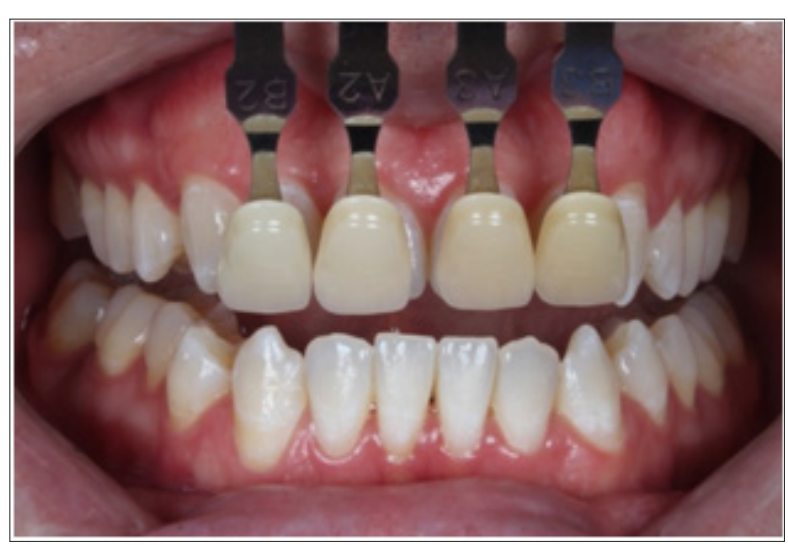

Figure 18: Final shade mapping - comparison to the inf. initial shade.

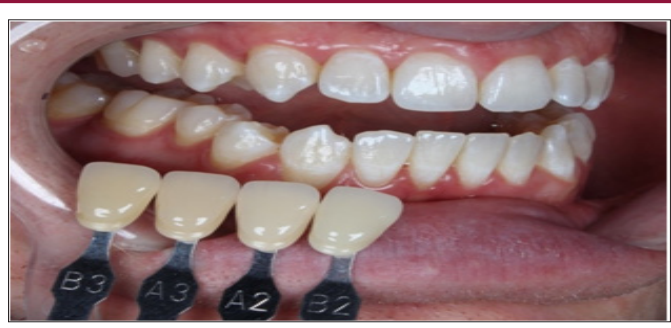

Figure 19: Final shade mapping - comparison to the right posterior initial shade.

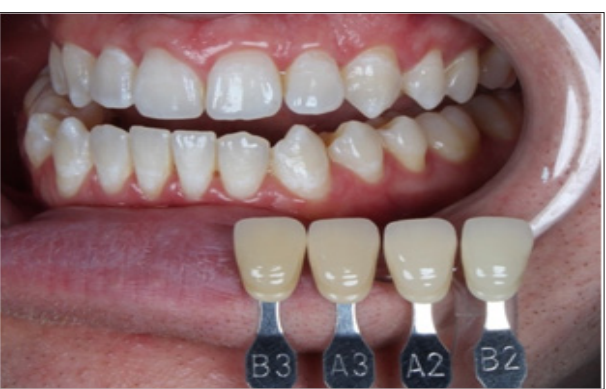

Figure 20: Final shade mapping - comparison to the left post initial shade.

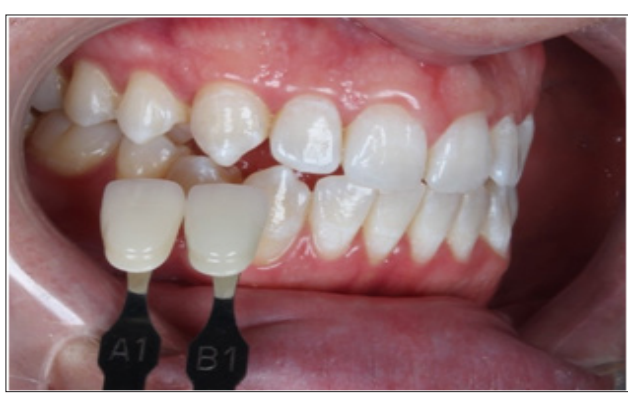

Figure 21: Final shade mapping - right posterior take.

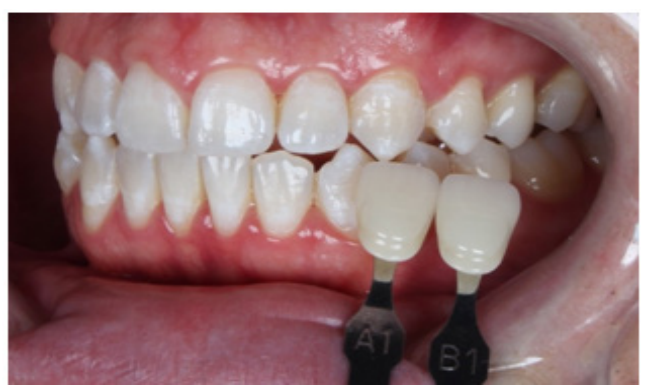

Figure 22: Final shade mapping - left posterior take.

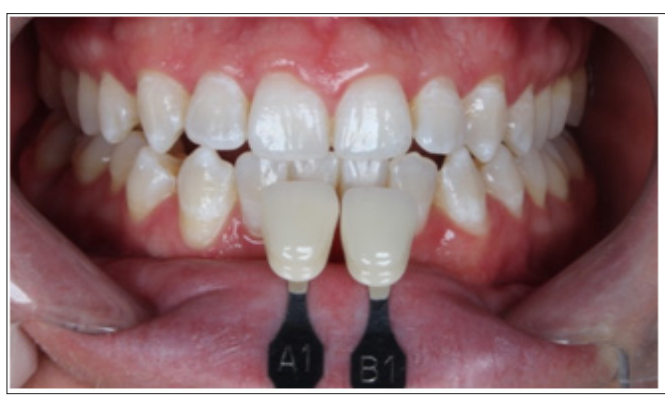

Figure 23: Final shade mapping - final overview take. 

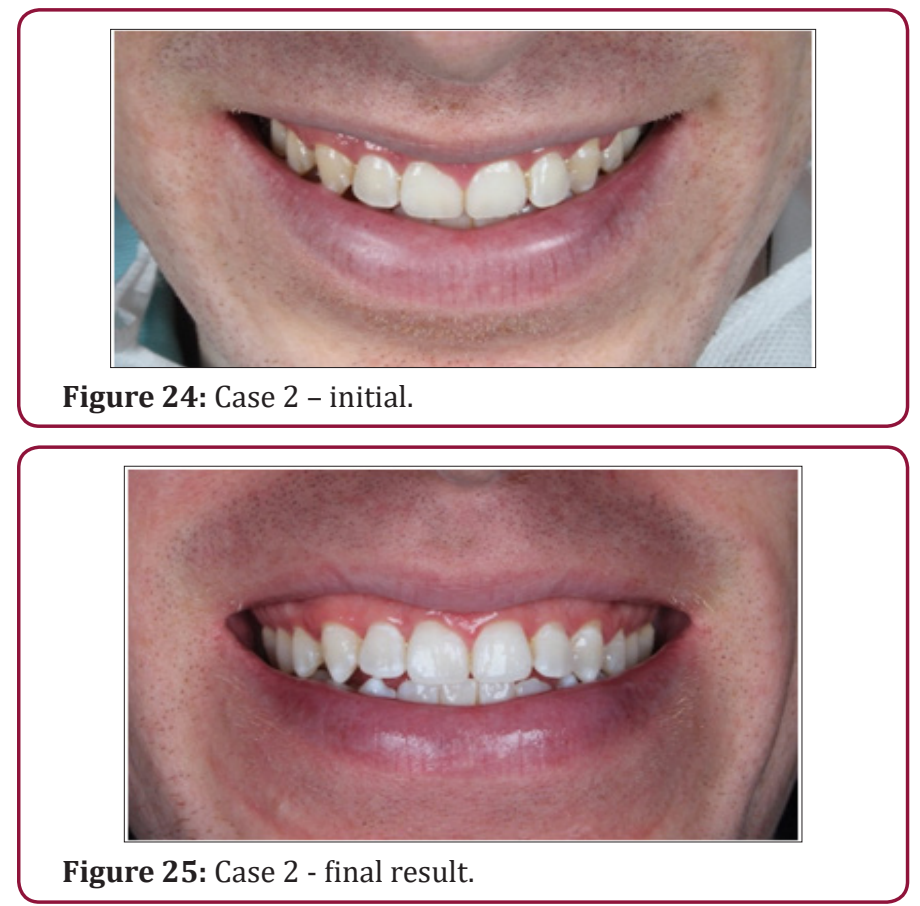

\section{Discussion}

Before starting the treatment, some guidelines are made to minimize or even avoid discomfort along the treatment, such as avoiding too hot and too cold foods and beverages, low $\mathrm{pH}$ foods and beverages, stop smoking (quitting is desirable) and every activity that can hyper stimulate tooth sensitivity. Both cases was used CP $10 \%$ in overnight technique, for at least six hours per application. That's because this substance in that concentration has shown minimum or none sensitivity along the research and clinical reports, positive results in a relatively short term $[7,8]$. According to ADA (American Dental Association), the overnight CP $10 \%$ dental bleaching is accepted as a safe technique, achieving the greatest benefit from the gel and requiring a shorter number of bleaching days (8). The initial standard conduct is to perform the treatment during the sleep time to avoid removing the tray to talk or drink, improving the chemical effects on teeth. Both patients went well using the tray overnight. But if they hadn't for any reason, they would have done the treatment awake, which means changing the $\mathrm{CP}$ concentration to a higher one and decreasing the usage time per application.

There are groups of teeth that respond faster than others. The incisors - superior and inferior - present a thinner structure, which facilitates the $\mathrm{CP}$ penetration and its chemical reaction. The canines and premolars are bulky compared to incisors. So the chemical reaction needs to be wider and deeper to reach all the pigment molecules, which means more bleaching time to match the incisors. As a complementary technique, the "in office" bleaching was discarded not just because of the positive response in both cases, but to avoid sensitivity. There are some situations that dentin does not respond so well to treatment as it should, especially in older patients, which present dentin sclerosis with deep and/or dark pigmentation. So, to improve the results, the "in office" bleaching operates strongly all over the enamel to make it less translucent, delivering a bleached shade and hiding part of the sclera dentin. Comparing case 1 to case 2, a speed response to dental bleaching was noticed. The patient of case 1 is older than the patient of case 2 . Young teeth have wider and patent dentinal tubules, crystalline and flexible collagen fibers and consequently present high permeability. Therefore, young teeth would favor ionic diffusion of the bleaching agent through dentine, increasing the chemical reaction making the patient 2 speed result faster than patient 1 (9). In results like of the patient 2, where teeth become lighter than the classic shade guide, another shade guide is used to compare lighter teeth called "bleach shade guide".

Until a few years ago, according to laboratorial studies, the aim conduct to avoid bad results was to prescribe the "white diet" to the patient during the treatment. The white diet consists in not eating and drinking anything that presents high levels of pigments, such as coffee, red wine, chocolate, condiments, soft drinks, dark juices, tomato sauce etc. But recent in vivo and in situ studies indicate that consuming all these food and drink is irrelevant to dental bleaching results $(5,6,7)$. About the in-office technique, in addition to higher sensitivity, another negative issue that should be considered is the color stability after treatment [9]. Studies have shown great loss of whitening result after 2 weeks, and this loss continued until week 10, returning to original teeth color. That is why the clinical recommendation is to use the in-office technique as a complement to the at home technique $(3,4)$.

\section{Conclusion}

Supported by clinical experience and scientific research, the authors believe to be the overnight CP $10 \%$ technique the best dental bleaching treatment available, which provides comfort along the treatment (and post-operative), color stability and long lasting result. Dental bleaching can be predicable, comfortable and safe with satisfying results provided it is performed correctly.

\section{References}

1. JE Dahl, U Pallesen (2003) Tooth Bleaching-A critical review of the biological aspects. Crit Rev Oral Biol Med 14(4): 292-304

2. FC Marson, LG Sensi, LC Vieira, E Araujo (2008) Clinical evaluation of In-Office dental bleaching treatments with and without the use of light activation sources. OpDent 33(1): 15-22.

3. D Dietschi, S Rossier, I Krejci (2006) In vitro colorimetric evaluation of the efficacy of various bleaching methods and products. Quintessence Int 37(7): 515-526.

4. BA Matis, MA Cochran, G Eckert (2009) Review of the effectiveness of various tooth whitening systems. Op Dent 34(2): 230-235.

5. BA Matis, G Wang, JI Matis, NB Cook, GJ Eckert (2015) White diet is it necessary during tooth whitening? Op Dent 40(3): 235-240.

6. M Rezende, AD Loguercio, A Reis, S Kossatz (2013) Clinical effects of exposure to coffee during at home vital bleaching. Op Dent 38(6): E229-E236.

7. AL Briso, TC Fagundes, MO Gallinari, J Moreira, L de Almeida, et al. (2016) An in situ study of the influence of staining beverages on color alteration of bleached teeth. Op Dent 41(6): 627-633.

8. BA Matis (2003) Tray whitening: what the evidence shows. Compendium 24(4A): 354-362. 
9. GP Lee, MY Lee, SOY Lum, RSC Poh, KC Lim (2004) Extraradicular diffusion of hydrogen peroxide and $\mathrm{pH}$ changes associated with intra coronal bleaching of discoloured teeth using different bleaching agents. Int Endod J 37(7): 500-506.

\section{(c) (i) This work is licensed under Creative}

Submission Link: http://biomedres.us/submit-manuscript.php

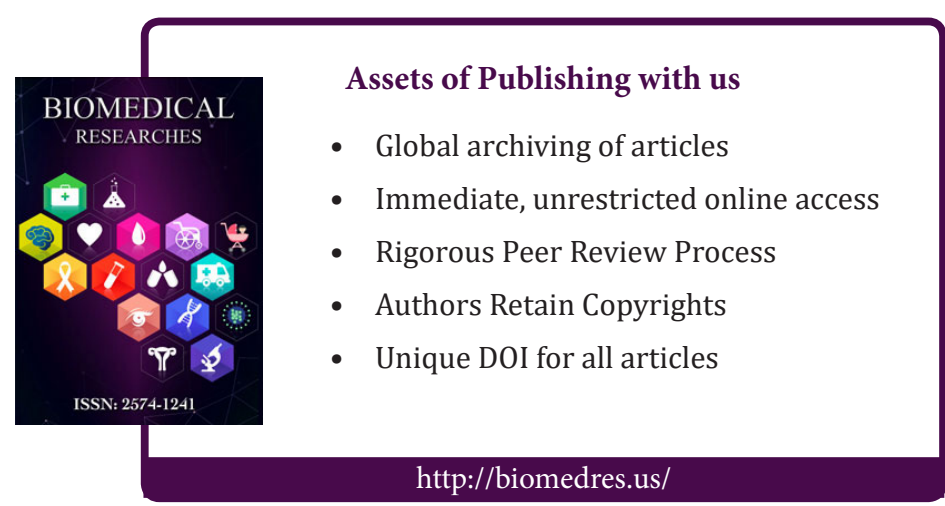

